\title{
Wireless Industrial Communication for Connected Shuttle Systems in Warehouses
}

\author{
Abdulkadir Karaagac, Jetmir Haxhibeqiri, Wout Joseph, Ingrid Moerman, Jeroen Hoebeke \\ Ghent University - imec, IDLab, Department of Information Technology \\ Technologiepark Zwijnaarde 15, B-9052 Ghent, Belgium \\ \{abdulkadir.karaagac,jetmir.haxhibeqiri,wout.joseph, ingrid.moerman, jeroen.hoebeke\}@ugent.be
}

\begin{abstract}
In the last decade, we have seen the uptake of connected solutions to support and improve manufacturing, warehousing and distribution operations in industry. However, deterministic and reliable wireless communication in largescale, dynamic and mobile applications still remains one of the greatest challenges for the realization of the Digital Factory of the Future. In this paper, we investigate more open, flexible and reliable wireless network structures that can be used in connected warehouses, focusing on connected shuttle systems for transporting goods.
\end{abstract}

Keywords - Industrial Networks, 802.15.4e, TSCH, 802.15.4 Sub-GHz, Warehouse Environment

\section{INTRODUCTION}

For many years, industrial companies have been constantly looking for ways to further automate processes and improve efficiency in pursuit of their business objectives. Beyond the design and production processes, warehousing and distribution operations are increasingly considered to be of strategic importance for obtaining a competitive advantage. Typically, mobile robots, such as automated guided vehicles (AGVs) or shuttle systems, are used to transport goods throughout the warehouse and across warehouse pallet racking systems by following navigation instructions coming from a central control server based on the current status and needs. Therefore, wireless connectivity with these mobile systems plays a key role in the realization of these automated and future-proof warehouse systems.

However, due to the dynamic and mobile nature of these applications, traditional wired and wireless communication technologies turned out not to be sufficient to meet strong communication requirements, especially in very harsh conditions of warehouses. For instance, wireless industrial standards, WirelessHart [1] and ISA100.11a [2], typically have difficulty coping with dynamic and mobile large-scale networks due to their centralized network management scheme [3]. In addition, these solutions also do not yet embrace the latest digital Internet of Things technologies and shift to more open protocols, hence limiting flexibility.

Recently, there has been a lot of research on introducing more open and flexible, but equally reliable networking technologies that can be used in industrial applications. For instance, the IEEE $802.15 .4 \mathrm{e}$ protocol is a recent MAC amendment of the IEEE 802.15.4 standard, specially designed for harsh industrial environments with a reliable and deterministic communication scheme based on TimeSlotted Channel Hopping [4]. Also, a recent IETF working group, named 6TiSCH, has been formed to investigate IPv6 connectivity over the TSCH mode of IEEE 802.15.4e protocol [5]. However, since most of these research efforts mainly focus on fixed networks with a static nature, the mobility support still remains one of the greatest challenges for wireless industrial networks at this point in time.
In the scope of this paper, we investigate communication solutions which can provide reliable and real-time connectivity for mobile intelligent self-contained transport vehicles, "shuttles", which move in 2 dimensions within the storing racks of warehouse to transport and store goods. For that purpose, we first examined the specific channel characteristics and challenges of the target environment via a measurement campaign in a real warehouse, where we measured the operational range and reliability of the considered technologies in the storing racks. Then, we design communication solutions and network architectures based on the previously mentioned networking technologies; $802.15 .4 \mathrm{e}$ and 6TiSCH. The centralized network management mechanisms will be used to bring the deterministic feature of industrial standards into an IPv6 context. On the other side, more dynamic self-configuring and self-healing networking mechanisms will be realized with the distributed scheduling (Scheduling Function 0 and 1) and routing mechanisms (RPL) defined in standards [5-6]. In addition, we also investigate the potential of 802.15.4 in the $868 \mathrm{MHz}$ frequency band to be used as networking solution for large scale warehouse applications with softer latency requirements.

Based on these technologies, we consider 3 communication schemes; (i) an $802.15 .4 \mathrm{e} 2.4 \mathrm{GHz}$ network with a cabled backbone, (ii) an $802.15 .4 \mathrm{e} 2.4 \mathrm{GHz}$ network with a wireless backbone and (iii) an $802.15 .4868 \mathrm{MHz}$ network with a star topology. In this work, we only describe the proposed system architectures and assess their potential to be used as a mobile wireless industrial networking solution, especially for the targeted use case. As a future work for this paper, we will analyze the performance of each approach and demonstrate its potential through extensive experiments on a wireless testbed for a variety of scenarios.

The remainder of this paper is organized as follows. In Section II, a detailed description of the target use case is provided. Section III presents the measurement campaign conducted to obtain the channel characteristics of a warehouse environment. Afterwards, Section IV discusses about our approach for network architecture and design. Finally, Section V concludes the paper.

\section{USE CASE : 2D ShUtTle System}

The considered use case is an automated warehouse system based on mobile intelligent self-contained transport vehicles, "shuttles", which can move in $2 \mathrm{D}$ within the storing racks of a warehouse and are able to store goods in a very compact way. The shuttles have to be battery powered due to their dynamic nature and they can be charged during their inactive periods. 
In order to accurately position and distribute the goods and interact with the operators, the shuttles have to be always connected to a central server via a reliable and realtime wireless communication infrastructure for time-critical status updates and order assignments to navigate in racks. An abstract model for the warehouse shuttle system is provided in Figure 1.

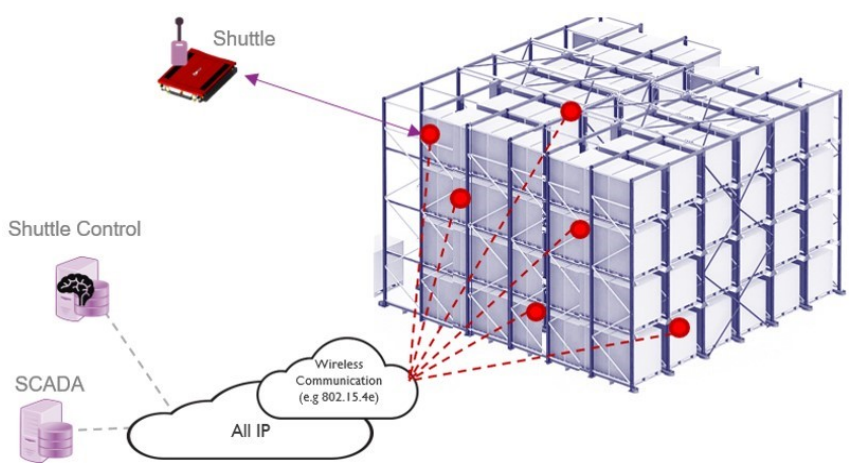

Fig. 1 2D Shuttle System Model in Warehouses

The warehouse environment is characterized by a metal racking structure and a dense storage of loads, possibly containing high percentages of liquids or metals. This creates a very harsh environment for radio waves to penetrate through. In addition, shuttle robots can drive at reasonably fast speeds up to $3 \mathrm{~m} / \mathrm{s}$ in 2D plain on a certain level of the pallet racking. Considering very dense storage of goods, this can result in very frequent, latency-sensitive handovers for the moving shuttles when using off-the-shelf wireless networks consisting of multiple access points, such as IEEE 802.11. Therefore, an optimal networking solution needs to be able to handle very frequent handovers softly without breaking overall network formation.

\section{ChanNEL MEASUREMENTS In A REAL WAREHOUSE}

The target communication solution has to be designed according to the specific characteristics and challenges of the environment. Therefore, before we focus on the architecture and design of the communication system, we performed a measurement campaign to analyze the channel characteristics and operational range for our target technologies $(802.15 .42 .4 \mathrm{GHz} \& 868 \mathrm{MHz})$, in a real warehouse environment. Based on the outcome of these measurements, we will design the medium access, routing and handover mechanisms for the target communication technologies. In addition, it can also be used to determine the backbone network and number of anchors needed for each technology for a certain area. The measurement plan, used equipment and measurement results are provided in the following sections.

\section{A. Measurement Plan}

\section{1) Environment}

The LeenBakker Warehouse, near Breda in the Netherlands, covers a total area of more than $15.000 \mathrm{~m} 2$. It consists of various sections including storage racks with multiple levels, receiving and shipping locations, aisles for AGVs, forklifts and cranes. Figure 2 shows one of the pallet racking zones where the pallets are automatically stored in horizontal rows with multiple levels. Figure 2 also represents the details and the dimensions of the testing area, where we operated our measurements. The blue corridors, in this plan, are the aisles for crane movement, whereas the remaining areas represent the racks that can be filled with goods.
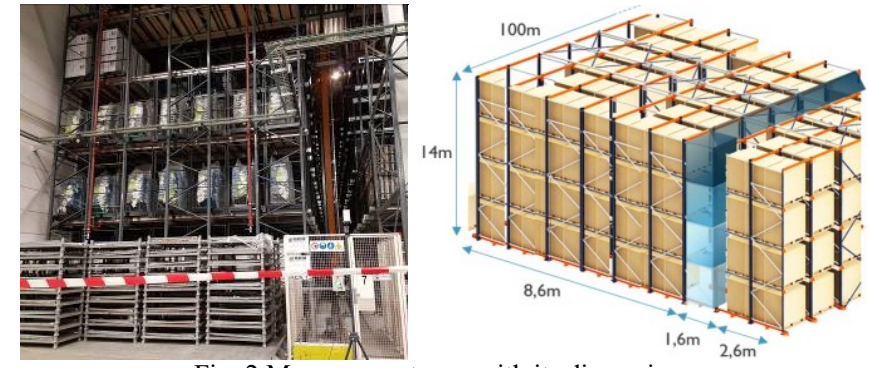

2)

Fig. 2 Measurement area with its dimensions

\section{Equipments}

For our measurements, we used a commercially available low power sensor device, Zolertia Remote [7], which has both an $802.15 .42 .4 \mathrm{GHz}$ and $868 \mathrm{MHz}$ radio. The technical specification of the device is provided in Table 1. As it is presented in Table 1, the 802.15.4 Sub-GHz radio has a higher transmission power and greater receiver sensitivity compared to the $2.4 \mathrm{GHz} 802.15 .4$ radio.

TABLE I. USED DEVICES FOR RANGE MEASUREMENTS

\begin{tabular}{clccc}
\hline Technology & $\begin{array}{l}\text { Device } \\
\text { Name }\end{array}$ & $\begin{array}{c}\text { Max Transmit } \\
\text { Power }\end{array}$ & $\begin{array}{c}\text { Antenna } \\
\text { Gain }\end{array}$ & $\begin{array}{c}\text { Receiver } \\
\text { Sensitivity }\end{array}$ \\
\hline $\begin{array}{c}802.15 .4 \\
(2.4 \mathrm{GHz})\end{array}$ & $\begin{array}{l}\text { Zolertia } \\
\text { Remote }\end{array}$ & $3 \mathrm{dBm}$ & $5 \mathrm{dBi}$ & $-97 \mathrm{dBm}$ \\
$\begin{array}{c}802.15 .4 \\
(868 \mathrm{MHz})\end{array}$ & $\begin{array}{l}\text { Zolertia } \\
\text { Remote }\end{array}$ & $14 \mathrm{dBm}$ & $5 \mathrm{dBi}$ & $-109 \mathrm{dBm} *$ \\
\hline & & & & $* 0 \mathrm{kbps}$
\end{tabular}

3) Measurement Setup

For each technology, we performed a similar measurement scheme with one transmitter fixed at a static point and one receiver node positioned at different points on different layers of the storing racks, as it is shown in Figure 3. After the transmitter and receiver are positioned on certain points, the transmitter starts to broadcast packets with a length of 40 and 125 bytes respectively. These packet sizes have been chosen in order to see the packet loss probability for larger data packets (125 bytes) and potential shorter acknowledgement packets (40 bytes). We collected the Received Signal Strength (RSSI) values for received packets and the Packet Success Rate (PSR) values for each position and each technology at the same time. For 802.15.4 at 2.4 $\mathrm{GHz}$, we collected data for 2 different channels, channel 15 and 26 , to see the possible contribution of the channel hopping mechanism to the communication performance.

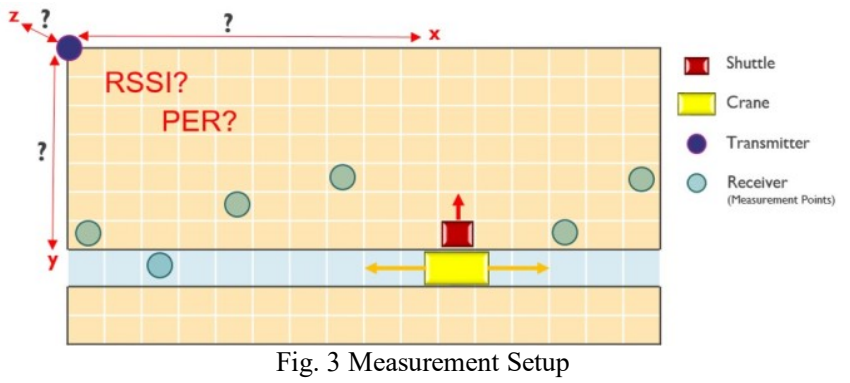

\section{B. Results}

In order to predict the operational range of the considered technologies, we initially measure and predict the path loss that the radio signals will encounter inside the storing racks as a function of the distance. To that end, we performed RSSI measurements while the receiver is positioned on a crane that moves with a constant speed until the end of the storing racks. Based on these measurements, we establish simple log-distance path loss models which can be used to predict the coverage for different transmission power 
configurations. The collected RSSI values at certain distances and constructed path loss models for these technologies are provided in Figure 4 and 5 respectively. For the $2.4 \mathrm{GHz}$ frequency band, we predicted a path loss exponent equal to 2.94 , whereas 3.1 for $868 \mathrm{MHz}$. Figure 4 shows that, with $3 \mathrm{dBm}$ transmission power, the RSSI values are becoming lower than the reception sensitivity for distances around 40 meters for the $802.15 .42 .4 \mathrm{GHz}$ technology. However, by using higher transmission powers, this theoretical range can be increased to longer distances. For instance, with $14 \mathrm{dBm}$ transmission power, it would be possible to achieve more than $80 \mathrm{~m}$ coverage in the same environment. According to the 802.15.4 standard [8] and existing radio chips [9-10], we can consider up to $20 \mathrm{dBm}$ transmission power for communication solutions using the 802.15.4 2.4GHz technology. On the other side, in Figure 5, the constructed path loss model shows that 802.15.4 $868 \mathrm{MHz}$ would still cover distances of $100 \mathrm{~m}$ with $0 \mathrm{dBm}$ transmission power.

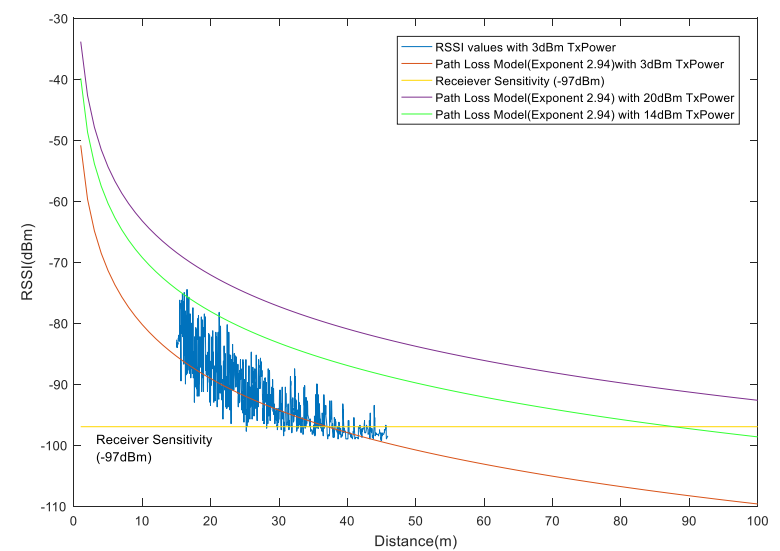

Fig. 4 RSSI values for 802.15.4 2.4 GHz and Path Loss Model

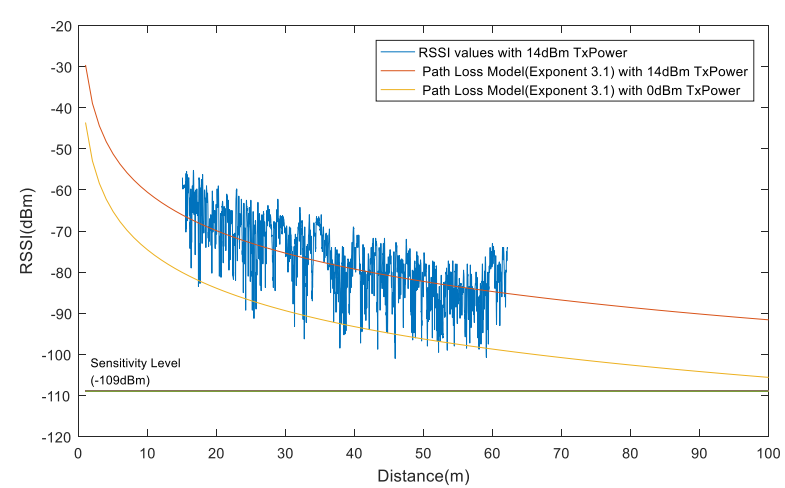

Fig. 5 RSSI values for 802.15.4 868 MHz and Path Loss Model
In addition to the path loss measurements, we also performed operational reliability and range measurements for various positions at different distances and levels. The collected PSR and RSSI values of 11 different positions and their location relative to transmitter is provided in Table 2. In this table, the distances between the transmitter and the receiver points are provided in terms of two different parameters. Beside the real distance, which is the total distance between the transmitter and receiver in $\mathrm{x}, \mathrm{y}$ and $\mathrm{z}$ plane, the level difference is also provided, because we observed that the existence of level differences has a stronger effect compared to the distance in meters in any direction.

From Table II, we can conclude that $2.4 \mathrm{GHz} 802.15 .4$ can only provide reliable connectivity till $15 \mathrm{~m}$ distance on the same level. However, after that point, the success rate drops below $40 \%$ with a RSSI value lower than $-90 \mathrm{dBm}$. On the other side, Position 6 and 7 show that the system is not able to provide reliable connectivity for neighbor levels even in the case of very short distances. Overall, the PSR values were in the range of $20 \%-40 \%$ for distances between 15 $30 \mathrm{~m}$ with changing RSSI values. The receiver became completely out of range after $40 \mathrm{~m}$ distance from the transmitter. Another important outcome of these results is the confirmation of the possible positive effect of channel hopping in such harsh environment. For instance, in Position 2 channel 26 provided a better performance compared to channel 15, whereas channel 15 performed remarkably better in Position 3.

For 802.15.4 Sub-GHz, we observed longer communication ranges with almost no packet loss even in the furthest point in the warehouse, which is more than $100 \mathrm{~m}$ away from the transmitter. $802.15 .4868 \mathrm{MHz}$ is able to use $11 \mathrm{dBm}$ more transmit power and $12 \mathrm{dBm}$ lower reception sensitivity compared to $802.15 .42 .4 \mathrm{GHz}$ technology, resulting in a $23 \mathrm{dBm}$ larger link budget. Also, since it is using a relatively low frequency, it can easier penetrate goods compared to $802.15 .42 .4 \mathrm{GHz}$. Considering the reception sensitivity level and the measured average RSSI value for the furthest point, we can predict that 802.15.4 $868 \mathrm{MHz}$ can even provide communication in larger warehouses with denser storages. As a downside, in this frequency band, the European regulations typically ask for adhering to a $1 \%$ duty cycle per sub-band or applying a "listen-before-talk and adaptive frequency agility" mechanism. In addition, the available bandwidth and thus number of channels is much more limited.

TABLE II. PACKET SUCCESS RATE AND SIGNAL RECEPTION STRENGTH FOR DifFERENT POSITIONS

\begin{tabular}{|c|c|c|c|c|c|c|c|c|c|c|c|}
\hline & \multirow{2}{*}{$\begin{array}{l}\text { Real } \\
\text { Distance } \\
(\mathrm{m})\end{array}$} & \multirow{2}{*}{$\begin{array}{c}\text { Level } \\
\text { Difference }\end{array}$} & \multicolumn{3}{|c|}{ 802.15.4 - Channel 15} & \multicolumn{3}{|c|}{ 802.15.4 - Channel 26} & \multicolumn{3}{|c|}{ 802.15.4 - 868MHz } \\
\hline & & & $\begin{array}{c}\text { PSR(\%) } \\
\text { 40B }\end{array}$ & $\begin{array}{c}\text { PSR(\%) } \\
125 B\end{array}$ & $\begin{array}{c}\text { RSSI } \\
\text { (dBm) }\end{array}$ & $\begin{array}{c}\text { PSR(\%) } \\
\text { 40B }\end{array}$ & $\begin{array}{c}\text { PSR(\%) } \\
125 B\end{array}$ & $\begin{array}{c}\text { RSSI } \\
\text { (dBm) }\end{array}$ & $\begin{array}{c}\text { PSR(\%) } \\
\text { 40B }\end{array}$ & $\begin{array}{c}\text { PSR(\%) } \\
125 B\end{array}$ & $\begin{array}{c}\text { RSSI } \\
\text { (dBm) }\end{array}$ \\
\hline Position1 & 8.4 & 0 & 96.28 & 96.20 & -75.57 & 97.24 & 97.84 & -76.70 & 100.00 & 100.00 & -59.67 \\
\hline Position2 & 11.1 & 0 & 80.80 & 63.16 & -89.84 & 97.80 & 98.00 & -89.08 & 100.00 & 100.00 & -62.75 \\
\hline Position 3 & 13.4 & 0 & 95.64 & 95.68 & -86.37 & 17.96 & 16.20 & -99.00 & 99.94 & 100.00 & -62.44 \\
\hline Position4 & 15.7 & 0 & 30.23 & 29.09 & -98.05 & 25.14 & 17.64 & -98.06 & 100.00 & 99.75 & -70.10 \\
\hline Position5 & 29.8 & 0 & 31.12 & 27.96 & -98.10 & 0.00 & 0.00 & -100.00 & 100.00 & 100.00 & -76.63 \\
\hline Position6 & 9.5 & 1 & 43.48 & 41.48 & -93.16 & 34.04 & 27.96 & -96.85 & 100.00 & 100.00 & -58.98 \\
\hline Position7 & 14.2 & 1 & 31.57 & 28.67 & -95.13 & 24.59 & 19.53 & -95.11 & 100.00 & 100.00 & -54.47 \\
\hline Position8 & 15.9 & 1 & 26.96 & 21.76 & -99.04 & 26.76 & 19.20 & -98.78 & 99.94 & 100.00 & -77.52 \\
\hline Position9 & 18.0 & 2 & 31.12 & 28.36 & -95.75 & 23.76 & 15.68 & -99.35 & 100.00 & 100.00 & -65.19 \\
\hline Position 10 & 12.6 & 4 & 29.49 & 25.13 & -93.63 & 24.14 & 16.72 & -94.91 & 100.00 & 100.00 & -49.78 \\
\hline
\end{tabular}




\section{SOLUTION DESIGN}

As described in the introduction, due to the insufficiency of wireless industrial standards to cope with the dynamic and mobile nature of large scale networks, we investigate the use of more flexible and configurable wireless networks in warehouse systems to meet the strong application requirements. Based on the previously mentioned technologies and the outcome of the measurement campaign, we consider three potential communication schemes; (i) $802.15 .4 \mathrm{e}$ with a cabled backbone, (ii) $802.15 .4 \mathrm{e}$ with a wireless backbone and (iii) an $802.15 .4868 \mathrm{MHz}$ network with a star topology.

\section{A. 802.15.4e with Wired Backbone}

Generally, 802.15.4 technology is used in multi-hop wireless sensor networks. To ease the realization in harsh environments, a wired and fixed network can be considered based on multiple border routers installed throughout the warehouse, resulting in a single hop 802.15 .4 e network from the perspective of the mobile shuttles as well as easier management and routing schemes. However, this system requires cabling for both power and communication. An abstract system model is presented in Figure 4.

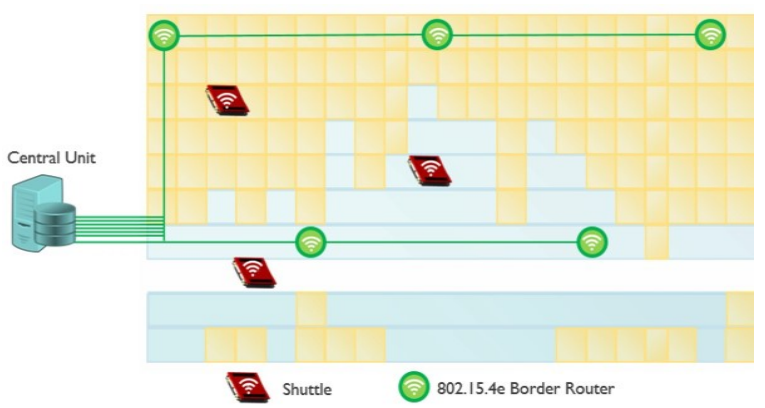

Fig. 4 Sample Architecture for 802.15.4e Network with Cabled Backbone

\section{B. 802.15.4e with Wireless Backbone}

This setup is targeting fully mesh, self-healing networks, resulting in the simplest installation and maintenance process. To be able to install such a system, we might only need to provide cabling for power or might only rely on batteries in some applications, which eases installation and maintenance. Figure 5 represents a sample system setup for 2D Shuttle system with 802.15.4e Network with Wireless Backbone.

The $802.15 .4 \mathrm{e}$ technology is not connection oriented (like Wi-Fi) and as such does not incur any handover latency. However, routing and scheduling under mobility is still a challenge that needs to be handled. Therefore, within this context, we focus our efforts on routing and scheduling techniques and network management mechanisms in order to achieve mobility support in wireless industrial networks.

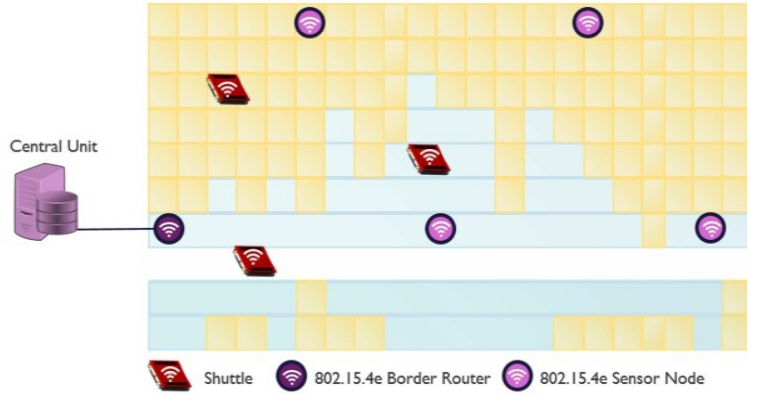

Fig. 5 Sample Architecture for 802.15.4e Network with Wireless Backbone

\section{C. $802.15 .4868 \mathrm{MHz}$ network with star topology}

The outcome of our measurement campaign shows that 802.15.4 868 MHz can provide a reliable and long-distance communication in warehouses. However, the duty-cycle requirements in $868 \mathrm{MHz}$ frequency band and increased interference limit the potential of this technology to be used in latency bounded deterministic networks. However, it can be a good alternative as a networking solution for large scale warehouse applications with softer latency requirements or it can also be used to obtain redundant links for other shorter range technologies.

\section{CONCLUSION}

This paper has presented realistic measurements regarding the channel characteristics of 802.15.4 technologies in a real warehouse environment. Next, three communication architectures are proposed, based on $802.15 .4 \mathrm{e} 2.4 \mathrm{GHz}$ and $802.15 .4868 \mathrm{MHz}$ Networks, to guarantee flexible and reliable connectivity in dynamic large-scale industrial applications, especially for connected warehouses. Current research focuses on the realization of those architecture, the provisioning of mobility support in $6 \mathrm{TiSCH}$ networks and the evaluation and demonstration of the proposed architectures through extensive experiments on a wireless testbed for a variety of scenarios.

\section{ACKNOWLEDGMENT}

HYCOWARE is a project realized in collaboration with imec. Project partners are Egemin, Aucxis and Intation, with project support from VLAIO (Flanders Innovation and Entrepreneurship).

\section{REFERENCES}

[1] IEC-62591, “Industrial communication networks - Wireless communication network and communication profiles-WirelessHART"

[2] IEC-62734, "Industrial networks - Wireless communication network and communication profiles - ISA 100.11a"

[3] P. Zand, S. Chatterjea, J. Ketema, P. Havinga1, "A Distributed Scheduling Algorithm for Real-time (D-SAR) Industrial Wireless Sensor and Actuator Networks", ETFA 2012

[4] "IEEE std. 802.15.4e, Part. 15.4: Low-Rate Wireless Personal Area Networks (LR-WPANs) Amendament 1: MAC sublayer", IEEE Computer Society, 2012

[5] M. R. Palattella, P. Thubert, X. Vilajosana, T. Watteyne, Q. Wang, and T. Engel, "6TiSCH wireless industrial networks: Determinism meets IPv6" in Internet of Things: Challenges and Opportunities., USA: Springer, 2014, pp. 111-141.

[6] T. Winter, P. Thubert, A. Brandt, J. Hui, R. Kelsey, P. Levis, K. Pister, R. Struik, J. P. Vasseur, R. Alexander "RPL: IPv6 Routing Protocol for Low-Power and Lossy Networks", RFC 6550, IETF, 2012

[7] "Zolertia Remote: Lightweight Internet of Things hardware development platform", http://zolertia.io/product/hardware/re-mote

[8] "IEEE Standard for Local and metropolitan area networks - Part. 15.4: Wireless Medium Access Control (MAC) and Physical Layer (PHY) Specifications for Low-Rate Wireless Personal Area Networks(LRWPANs)", IEEE Computer Society, 2011

[9] "EMB-Z2530PA: 2.4 GHz ZigBee-ready and 802.15.4-ready module", retrieved March 27, 2017, from http://www.embit.eu/products/wireless-modules/emb-z2530pa/

[10] Atmel Corporation, "ZigBit ${ }^{\mathrm{TM}} 2.4 \mathrm{GHz}$ Amplified Wireless Modules ATZB-A24-UFL/U0, Datasheet”, 2009 